УДК 544.344.015

DOI: $10.17308 / \mathrm{kcmf} .2019 .21 / 1159$

Поступила в редакцию 19.07.2019

Подписана в печать 15.08.2019

\title{
АНАЛИЗ ВЛИЯНИЯ ТЕМПЕРАТУРНОЙ ЗАВИСИМОСТИ ПАРАМЕТРОВ ДИФФУЗИИ НА ХАРАКТЕР РОСТА СЛОЕВ В ДВУХКОМПОНЕНТНОЙ МНОГОФАЗНОЙ СИСТЕМЕ
}

\author{
(2)19 Л. А. Молохина ${ }^{1}$, С. А. Филин ${ }^{2 \bowtie}$ \\ ${ }^{1}$ ул. Кибальчича, д. 2, корп. 4, кв. 86, 129164 Москва, Российская Федерация \\ ${ }^{2}$ Российский экономический университет им. Г.В. Плеханова \\ Стремянный переулок, 36, 117997 Москва, Российская Федерация
}

\begin{abstract}
Аннотация. Целью статьи является разработка феноменологической математической модели формирования и роста фаз в части влияния температурной зависимости параметров диффузии на характер роста слоев в двухкомпонентной многофазной системе. Темой исследования является анализ влияния температурной зависимости параметров диффузии на изменение характера роста слоев в двухкомпонентных многофазных системах. Предложено решение задачи использования температурного режима процесса диффузии при разработке технологических процессов сварки, пайки, нанесении покрытий и других, при которых в диффузионной зоне образуются интерметаллические слои, карбиды, нитриды, субоксиды, фосфиды и т. п. с заданными и контролируемыми эксплуатационными характеристиками получаемых новых материалов, их соединений, покрытий и пр. Результаты решения задачи позволяют по известным параметрам температурного режима процесса диффузии, полученным при исследовании двухкомпонентной многофазной системы, целенаправленно контролировать динамику роста, состав образующихся в процессе диффузии слоев, и их выходные параметры в данной системе для получения новых материалов с заданными свойствами.
\end{abstract}

Ключевые слова: температурная зависимость диффузии, фаза, межфазная граница, многофазная система, математическая модель, компонент.

\section{ВВЕДЕНИЕ}

В статьях $[1,2]$ в рамках разработки новой феноменологической теории взаимной диффузии предложена математическая модель роста фаз в двухкомпонентных многофазных системах при изотермическом отжиге и проведен анализ их роста в зависимости от соотношения параметров диффузии в двух соседних фазах.

Новая феноменологическая теория взаимной диффузии для двухкомпонентных многофазных систем учитывает, что согласно 2-му закону Фика параболической зависимости от времени диффузии подчиняется количество компонента, переходящего каждую межфазную границу через единицу площади, а не толщина слоя растущей фазы. Это приводит к более корректному расчету температурной зависимости параметров диффузии с учетом взаимного влияния на них процессов диффузии в двух соседних фазах. Совместный анализ графиков темпера-

$\bar{\triangle}$ Филин Сергей Александрович, e-mail: Filin.SA@rea.ru турной зависимости $\ln B_{i}=f(1 / T)$ и $\ln B_{i+1}=f(1 / T)$ от температуры отжига для двух соседних фаз: предыдущей $i$ и последующей $i+1$ показывает, как изменяется характер роста фазы $\boldsymbol{i}$ после появления на ее границе с компонентом $B$ новой фазы $(i+1)$.

Зависимость толщины слоя компонента $A$, пошедшего на рост фазы $i$ при переходе через межфазную границу раздела фаз « $(i+1)-i »$, и всех последующих $(n-i)$ фаз, образовавшихся в диффузионной зоне за исследуемый период диффузии, определяется уравнением [1, 2]:

$$
\sum_{i}^{n} h_{i}=\sqrt{B_{i}\left(t_{i}^{d}-\sum_{i=1}^{u} t_{i}^{u}\right)}
$$

где $\sum_{\mathrm{i}=1}^{n} h_{\mathrm{i}}$ - толщина слоя компонента $A$, пошедшая на рост фазы $i$ и всех последующих $(n-i)$ фаз, образовавшихся в системе к соответствующему периоду; $B_{i}$ - коэффициент скорости перехода компонента $A$ через каждую межфазную

Контент доступен под лицензией Creative Commons Attribution 4.0 License.

The content is available under Creative Commons Attribution 4.0 License. 
границу, равный тангенсу угла $\beta_{i}$ наклона прямой зависимости $h^{2}=f(\tau) ; \sum_{i=1}^{u} \tau^{u}-$ суммарный инкубационный период, предшествующий появлению фазы $i$ и отсчитываемый от начала изотермического отжига; $\tau^{d}$ - время изотермического отжига (диффузии).

Уравнение (1) показывает, что 2-й закон Фика описывает именно зависимость количества компонента $A$, переходящего в процессе диффузии каждую межфазную границу. При этом в уравнении (1) учтены длительности инкубационных периодов, предшествующие началу роста каждой фазы. Рост же фазы $(i+1)$ происходит за счёт частичного распада фазы $i$ на границе раздела «фаза $i$ - фаза $(i+1) »$, что приводит к появлению «перегиба» на графике роста фазы $i$ и к дальнейшему изменению характера роста фазы $i$ после появления в диффузионном слое фазы $(i+1)[1,2]$ :

$$
x_{i}=\sqrt{A_{i}\left(t_{i}^{d}-\sum_{i=1}^{u} t_{i}^{u}\right)}-\frac{c_{i+1}}{c_{i}} \sqrt{A_{i+1}\left(t_{i+1}^{d}-\sum_{i=1}^{u+1} t_{i+1}^{u}\right)},
$$

где $x_{i}$ - толщина слоя фазы $i ; A_{i}$ - коэффициент скорости роста фазы $i ; A_{i+1}$ - коэффициент скорости роста фазы $(i+1) ; C_{i}$ - средняя атомарная концентрация компонента $A$ в фазе $i$; $C_{i+1}$ - средняя атомарная концентрация компонента $A$ в фазе $(i+1) ; \sum_{i=1}^{u} \tau^{u}$ - суммарный инкубационный период, предшествующий появлению фазы $i$ и отсчитываемый от начала изотермического отжига; $\sum_{i=1}^{u+1} \tau^{u}$ - суммарный инкубационный период, предшествующий появлению фазы $(i+1)$ и отсчитываемый от начала изотермического отжига; $\tau^{d}$ - время изотермического отжига (время диффузии).

Анализ литературы по диффузии в многофазных системах показал, что для многих из них наблюдаются отклонения как в скорости роста фаз, так и в порядке появления интерметаллических фаз в диффузионном слое, аналогичный решению уравнения (2). В работах [3-8] отмечается отклонение динамики роста фаз от предсказываемой 2-м законом Фика параболической зависимости от времени диффузии, в других - несоответствие в расположении и количестве фаз на равновесных диаграммах состояния, выявляемых при изотермическом сечении диаграммы фазового состояния соответствующих систем. При этом скорость роста фаз в разных системах может весьма непредсказуемым образом увели- чиваться или замедляться как при изотермической выдержке, так и при изменении температуры процесса [7, 9-13]. В частности, известны системы, где первыми растут фазы с большей теплотой образования, чем предыдущая, или скорость роста фаз от времени диффузии не только не подчиняется параболической зависимости, но появившиеся первыми фазы могут потом исчезнуть в процессе роста следующей фазы [1, 2, 5, 6, 9-11, 13-18]. Причём на кинетических кривых роста слоев отмечается инкубационный период, в течение которого диффузионные слои не появляются [1-4, 6, 7, 9, 19-21].

Наличие перегиба на графике (рис. 1) роста 1-й фазы впервые отметил В. З. Бугаков [16] для системы «медь - кадмий», в которой рост $\varepsilon$-фазы при начале роста $\gamma$-фазы демонстрирует перегиб и изменение скорости роста є-фазы. Он предположил, что появление данного перегиба связано с изменением граничных условий для $\varepsilon$-фазы в период начала роста $\gamma$-фазы.

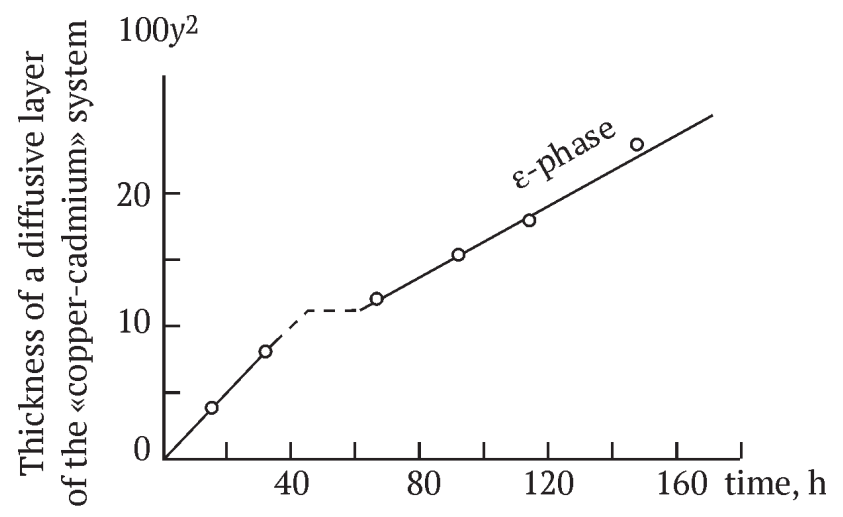

Рис.1 Рост диффузионного слоя системы «медь- кадмий»

[Fig. 1. Growth of a diffusive layer of the "copper-cadmium" system]

Цель исследования - разработка феноменологической математической модели формирования и роста фаз в части влияния температурной зависимости параметров диффузии на характер роста слоев в двухкомпонентной многофазной системе.

\section{ТЕОРЕТИЧЕСКИЙ АНАЛИЗ}

Температурная зависимость параметров диффузии $A_{i}, B_{i}$ и $D_{i}$ в двухкомпонентной многофазной системе. Повышение температуры изотермического отжига приводит к большей подвижности атомов как в структуре (например, в кристаллических решетках) самих взаимодействующих компонентов и растущих 
фаз, так и на разделяющих их межфазных границах. Определенный уровень энергии активации диффузии, необходимый для преодоления межфазного потенциального энергетического барьера на границе раздела и роста фаз, обеспечивает переход атомов из одного положения в другое вследствие непрерывного обмена кинетической энергией с соседними атомами, при этом уровень энергии активации диффузии возрастает с увеличением температуры процесса диффузии.

Эмпирическую зависимость константы скорости химической реакции $k$ от температуры установил С. Аррениус в 1889 г.:

$$
k=k_{0} \cdot \exp \left[-\frac{E_{a}}{R T}\right]
$$

где $k$ - константа скорости реакции; $k_{0}$ - постоянный множитель (предэкспонента); $E_{a}-$ энергия активации; $R$ - универсальная газовая постоянная $(R=8.31 \mathrm{~J} /(\mathrm{mol} \cdot \mathrm{K})) ; T-$ абсолютная температура, К. При этом закон Аррениуса применим и для определения уровня энергий активации в диффузионных процессах, так как показывает хорошее совпадение с экспериментальными данными в большинстве исследованных многофазных систем [4-6].

Для построения графика зависимости $\ln k=f(1 / T)$ (рис. 2) часто используют закон Аррениуса в логарифмической форме:

$$
\ln k=\ln A-\frac{E_{a}}{R T},
$$

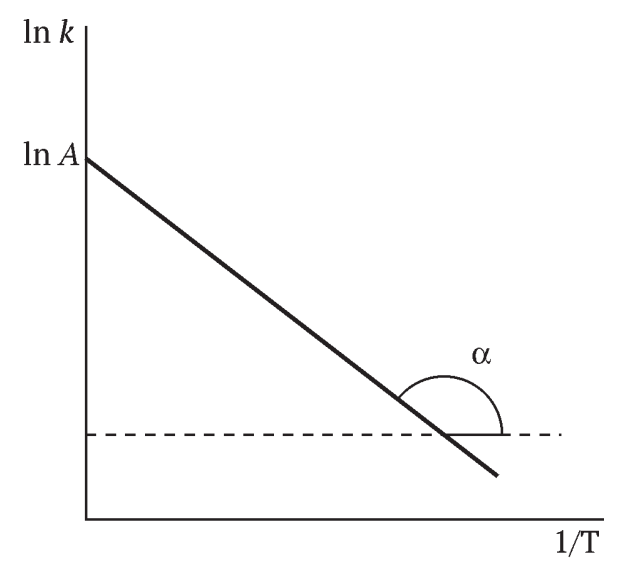

Рис. 2. Зависимость логарифма константы скорости химической реакции от величины, обратной температуре $(1 / T)$

[Fig. 2. Dependence of a logarithm of a constant of speed of chemical reaction on size, return temperature $(1 / T)]$ где $\ln A$ - соответствует точке пересечения графика зависимости $\ln k=f(1 / T)$ с осью ординат; тангенс угла $\alpha$, пропорционального энергии активации процесса диффузии:

$$
\operatorname{tg} \alpha=-\frac{E_{a}}{R T},
$$

В соответствии с законом Аррениуса зависимость всех параметров диффузии (коэффициентов взаимной диффузии $\left(D_{i}\right)$, скорости роста фаз $\left(A_{i}\right)$ и перехода компонента $A$ межфазных границ $\left.\left(B_{i}\right)\right)$ подчиняются экспоненциальному закону от температуры отжига:

$$
\begin{aligned}
& D_{i}=D_{0 i} \cdot \exp \left[-\frac{Q_{i}}{R T}\right], \\
& A_{i}=A_{0 i} \cdot \exp \left[-\frac{E_{i}}{R T}\right], \\
& B_{i}=B_{0 i} \cdot \exp \left[-\frac{G_{i}}{R T}\right],
\end{aligned}
$$

где $D_{i}$ - коэффициент взаимной диффузии; $A_{i}-$ константа скорости роста фазы, пропорциональная коэффициенту взаимной диффузии в фазе $i$; $B_{i}-$ коэффициент скорости перехода компонента $A$ через каждую межфазную границу, равный тангенсу угла $\beta_{i}$ наклона прямой зависимости $h^{2}=f(\tau) ; D_{0 i}, A_{0 i}, B_{0 i}$ - предэкспоненциальные множители; $Q_{i}$ - энергия активации взаимной диффузии в фазе $i ; E_{i}$ - энергия активации роста фазы $i ; G_{i}$ - энергия активации перехода межфазных границ.

Для построения графиков температурной зависимости параметров диффузии запишем уравнения (6-8) в логарифмической форме:

$$
\begin{aligned}
& \ln D_{i}=\ln D_{0 i}-\frac{Q_{i}}{R T}, \\
& \ln A_{i}=\ln A_{0 i}-\frac{E_{i}}{R T}, \\
& \ln B_{i}=\ln B_{0 i}-\frac{G_{i}}{R T},
\end{aligned}
$$

В большинстве исследований рост слоя каждой фазы подчиняется параболическому закону, полученному решением уравнения Мотано с учётом длительности инкубационного периода [16]:

$$
x_{i}^{2}=2 \frac{\Delta c_{i}}{c_{i}\left[1-c_{i}\right]} D_{i}\left(t^{d}-\sum_{i=1}^{i} t_{i}^{u}\right)=A_{i}\left(t^{d}-\sum_{i=1}^{i} t_{i}^{u}\right),
$$

где $\tau^{d}$ - время изотермического отжига (время диффузии).

Однако в уравнении (12) не учитывается влияние на скорость роста фаз в многофазном слое 
распада части слоя фазы $i$ на границах раздела фаз «i- $(i+1) »$ на рост фазы $(i+1)[1,2]$. Это приводит к ошибкам в расчетах параметров диффузии $A, B$ и $D$. В результате графики зависимости $\ln k=f(1 / T)$ имеют отклонения от прямой линии $[4,7,20,21]$.

Учет в уравнении (2) 2 -го члена
$\frac{c_{i+1}}{c_{i}} \sqrt{A_{i+1}\left(t_{i+1}^{d}-\sum_{i=1}^{u+1} t_{i+1}^{u}\right)}$, определяющего уменьшение толщины слоя фазы $i$, расходуемого на рост фазы $(i+1)$, приводит к более корректному расчету параметров диффузии $A_{i}, B_{i}$ и $D_{i}$. Эти параметры связаны между собой (без учёта влияния на толщину слоев разницы в плотности упаковки атомов в кристаллических решетках как самих исходных компонентов, так и всех фаз системы) следующими соотношениями:

$$
\begin{gathered}
x_{i} C_{i}=h_{i}, \\
A_{i}=2 \frac{\Delta c_{i}}{c_{i}\left[1-c_{i}\right]} D_{i}, \\
B_{i}=A_{i} C_{i}^{2}, \\
D_{i}=\frac{c_{i}\left[1-c_{i}\right]}{2 \Delta c_{i}} A_{i},
\end{gathered}
$$

где $h_{i}$ - толщина слоя компонента $A$, пошедшего на рост слоя фазы $i ; \Delta C_{i}$ - разница атомарных граничных концентраций компонента $A$ в фазе $i$; $C_{i}$-средняя атомарная концентрация компонента $A$ в фазе $i$.

\section{ЭКСПЕРИМЕНТАЛЬНАЯ ЧАСТЬ}

Анализ влияния температурной зависимости параметров диффузии на характер роста слоев в двухкомпонентной многофазной системе. Используем при построении графиков коэффициент скорости перехода компонента $A$ через каждую межфазную границу $B_{i}$, равный тангенсу угла $\beta_{i}$ наклона прямой зависимости $h^{2}=f(\tau)$. Коэффициенты $D_{i}$ и $A_{i}$ связаны с $B_{i}$ соотношениями (13-16).

Проанализируем совместно графики (рис. 3) температурной зависимости $\ln B_{i}=f(1 / T)$ и $\ln B_{i+1}=f(1 / T)$ от температуры отжига для двух соседних фаз: предыдущей $i$ и последующей $(i+1)$.

Как видно из рис. 3 , графики $\ln B_{i}=f(1 / T)$ и $\ln B_{i+1}=f(1 / T)$ могут быть параллельны (рис. $3 b$ ) или наклонными друг к другу (рис. 3a и 3c). При изменении температуры изотермического отжига в фазах $i$ и $(i+1)$ пропорционально изменяются и соотношения: $\left(B_{i}>B_{i+1}\right),\left(B_{i}=B_{i+1}\right)$ и $\left(B_{i}<B_{i+1}\right)$.

В статье [2] подробно рассмотрен вопрос об изменении характера роста предыдущей фазы $i$ при появлении на ее границе в компонентом $B$ следующей фазы $(i+1)$ при разных соотношениях параметров диффузии в фазах $A_{i}, B_{i}, D_{i}, A_{i=+1}$ $B_{i+1}$ и $D_{i+1}$. В статье показано, что характер роста фазы $\boldsymbol{i}$ зависит не только от энергий активации в каждой фазе, а от соотношения этих энергий в обеих фазах $i$ и $(i+1)$.

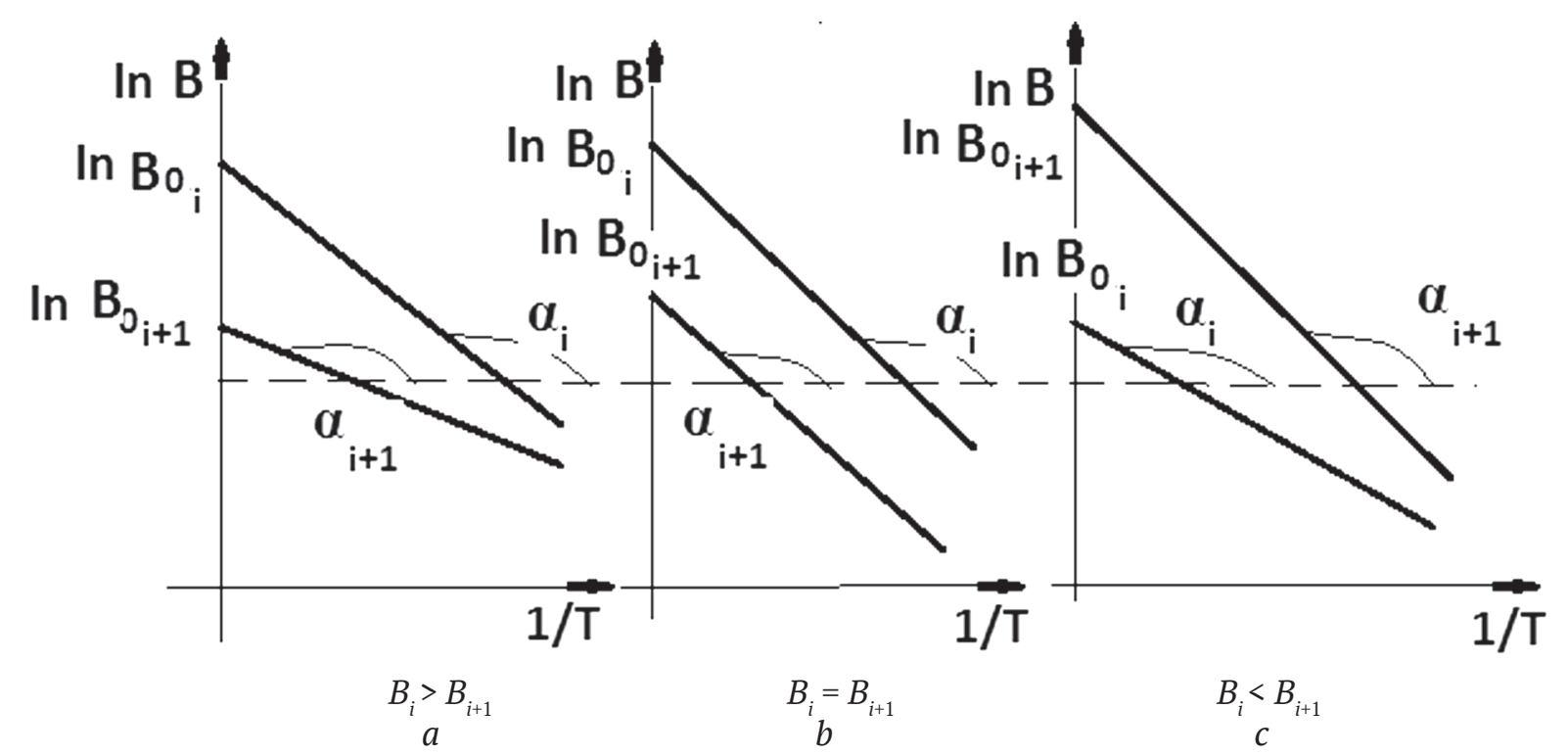

Рис. 3. Графики зависимости коэффициентов скорости перехода компонента $A$ через межфазные границы от обратной температуры для фаз $i$ и $(i+1)$

[Fig. 3. Schedules of dependence of coefficients of speed of transition of component $A$ through interphase borders from inverse temperature for phases $i$ and $(i+1)$ ] 
C ростом или снижением температуры отжига и, соответствующим изменением $A_{i}, B_{i}, D_{i}$, $A_{i=+1} B_{i+1}$ и $D_{i+1}$ будет изменяться и характер роста фазы $i$.

Построим графики температурной зависимости коэффициента скорости перехода компонента $A$ через межфазные границы $\ln B_{i}=f(1 / T)$ и $\ln B_{i+1}=f(1 / T)$ для роста фаз $i$ и $(i+1)$ (рис. 4). Рассмотрим случаи $(a)$ и $(b)$, когда прямые графиков пересекаются в зоне исследуемых температур при температуре перехода $T_{t}$.

Как видно из графиков (рис. 4), чем дальше отстоит $T_{\text {отжига }}$ от $T_{\mathrm{t}}$, тем больше разница коэффициентов $B_{i}$ и $B_{i+1}$ и тем больше разница в характере роста фазы $i$ при разных температурах.

Решая систему уравнений (9-11), рассчитываем температуру перехода $T_{\pi}$ :

$$
\begin{aligned}
T_{t . B_{i}}= & \frac{\left(G_{i+1}-G_{i}\right)}{R \ln \frac{B_{0 i+1}}{B_{0 i}},} \\
T_{t . B_{i}}= & T_{t . D_{i}}=T_{t . A_{i}} .
\end{aligned}
$$

Проведенный в работе [2] в соответствии с уравнениями (1) и (2) анализ случаев роста фаз в двухкомпонентной многофазной системе показал, что изменение характера роста фазы $i$ зависит от соотношения параметров диффузии в двух соседних фазах. Рост предыдущей фазы $i$ зависит от уровня энергии активации процесса диффузии, скорости роста следующей фазы $(i+1)$, суммарной длительности их инкубационных периодов и концентрации компонента $A$ в соседних фазах. Причём на разницу средних концентраций компонента $A$ в соседних фазах оказывает влияние как ширина области гомогенности этих фаз, так и ширина двухфазной области между ними.

На графиках (рис. 5 и 6) роста фазы $i$ после точки «перегиба» происходит замедление роста фазы $i$. Форма и размер «перегиба» зависят от соотношения всех параметров диффузии в двух соседних фазах. Далее фаза $i$ может: а) вновь продолжить рост, но со скоростью меньшей, чем это было до момента появления на её границе раздела с компонентом $B$ фазы $(i+1)$; b) практически не изменяться по толщине; c) продолжать уменьшаться, вплоть до полного исчезновения.

Проанализируем изменение характера роста фазы $i$ (рис. 5 и 6) при изменении температуры отжига $T_{0}$ в зависимости от соотношения энергий активации перехода межфазных границ $G_{i}$ и $G_{i+1}$ (рис. 4) для фаз $i$ и $(i+1)$.

Для случаев, когда энергия активации перехода компонентом $A$ межфазных границ
$\left(G_{i}>G_{i+1}\right)$ и при проведении отжига при темпеpaтурах выше $\left(T_{0}>T_{n}\right)$ (зона 1 - рис. $\left.4 a\right)$, параметр $\left(B_{i}>B_{i+1}\right)$. На рис. 5 [2] показано изменение характера роста фазы $i$ после появления на ее границе с компонентом $B$ фазы $(i+1)$. По мере снижения температуры отжига и уменьшения разницы между параметрами $B_{i}$ и $B_{i+1}$ скорость роста фазы $i$ снижается (рис. $5 a-5 c$ ) [2].

Для случаев, когда энергия активации перехода компонентом $A$ межфазных границ $\left(G_{i}<G_{i+1}\right)$ и при проведении отжига при температурах выше $\left(T_{\mathrm{o}}>T_{\text {п }}\right)$ (зона 1 - рис. $\left.4 b\right)$, параметр $\left(B_{i}<B_{i+1}\right)$. В этом случае, характер роста фазы $i$ соответствует рис. $6 b$ и $6 c$. При высоких температурах фаза $i$ может вообще не обнаруживаться в диффузионной зоне. И только по мере снижения температуры отжига и сближения значений $B_{i}$ и $B_{i+1}$, а также при переходе температур отжига в зону 2 (рис. $4 b)$ и достижении значений $\left(B_{i}>B_{i+1}\right)$ фаза $i$ будет обнаруживаться в диффузионном слое (рисунок $6 a-c$ ) [2].

\section{РЕЗУЛЬТАТЫ И ИХ ОБСУЖДЕНИЕ}

Из приведенных примеров видно, как изменяется характер роста предыдущей фазы $i$, после появления на ее границе с компонентом $B$ следующей фазы $(i+1)$. В работе [22] описан аналогичный случай роста фазы « $\mathrm{UAl}_{2}$ » после по-

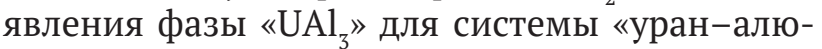
миний». При температурах < $560{ }^{\circ} \mathrm{C}$ фаза « $\mathrm{UAl}_{2} »$ увеличивается по толщине, а выше этой температуры - уменьшается (рис. 6a). Для объяснения этого явления и на основании решения уравнений (19-21) авторы работы [22] ввели в оборот некую «отрицательную» энергию активации роста фаз в данной системе:

где

$$
\begin{gathered}
E_{i}=\frac{4}{A_{i}}\left\{\left[\frac{(D K M)_{(i+1)_{1}}-(D K M)_{i_{2}}}{c_{i_{2}}-c_{(i+1)_{1}}}-\right.\right. \\
\left.\left.-\frac{(D K M)_{i_{1}}-(D K M)_{(i+1)_{1}}}{c_{(i+1)_{1}}-c_{i_{1}}}\right]\right\}, \\
M_{l m}=\left[Q_{i}+\frac{1}{k} \sum_{j+1}^{n} D_{j} Q_{j} \frac{d k}{d Q_{j}}\right] l m, \\
K=\sqrt{\tau} \frac{d c}{d x}\left(x, \tau, D_{i}, \ldots, D_{n}\right) .
\end{gathered}
$$

Однако данное явление легко объяснимо и без привлечения «отрицательной» энергии активации роста фазы. Кроме этого, решение уравнений (17-18) позволяет рассчитать температуру перехода, после которой характер роста фаз кардинально изменяется. 

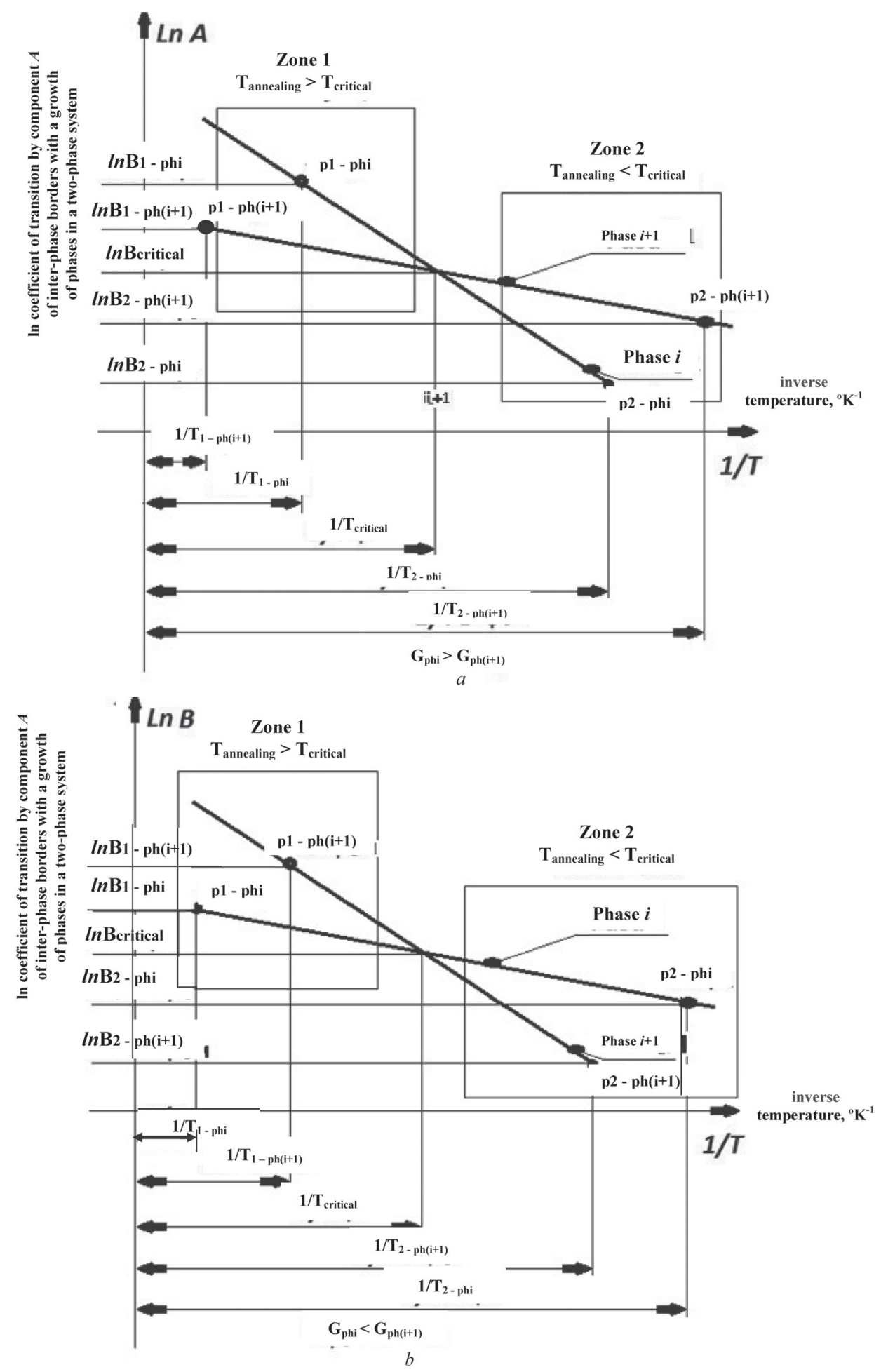

Рис. 4. Зависимость от обратной температуры коэффициента перехода компонентом $A$ межфазных границ при росте фаз в двухфазной системе, где $(a)$ - энергия активации фазы $(i+1)$ ниже энергии активации фазы $i ;(b)$ - энергия активации фазы $(i+1)$ выше энергии активации фазы $i$; $1, \mathrm{p} 2$ - точки 1,2 соответственно; $\operatorname{phi}, \operatorname{ph}(i+1)$ - фазы $i,(i+1)$ соответственно; $T_{\text {critical }}$ - критическая температура

[Fig. 4. Dependence on inverse temperature of coefficient of transition by component $A$ of inter-phase borders with a growth of phases in a two-phase system where $(a)$ - energy of activation of a phase $(i+1)$ is lower than energy of activation of phase $i ;(b)$ - energy of activation of a phase $(i+1)$ is higher than energy of activation of phase $I ; \mathrm{p} 1, \mathrm{p} 2$ are points 1 , 2, respectively; phi, ph $(i+1)$ are phases $i,(i+1)$, respectively; $T_{\text {critical }}$ - critical temperature] 


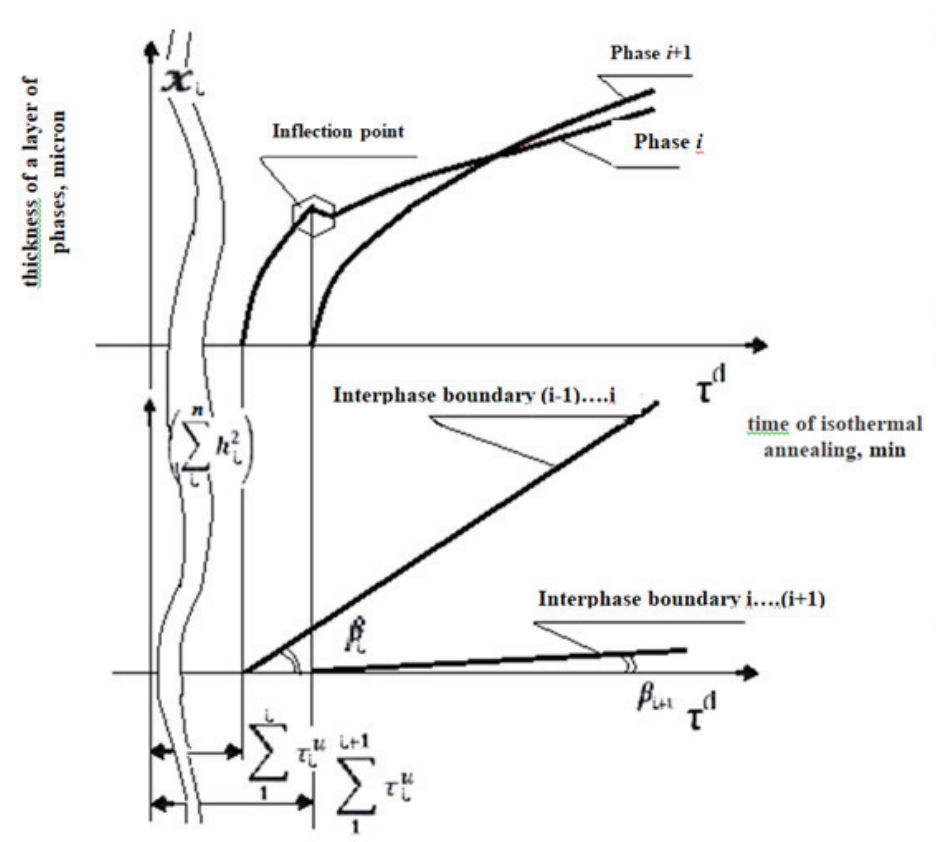

$a$

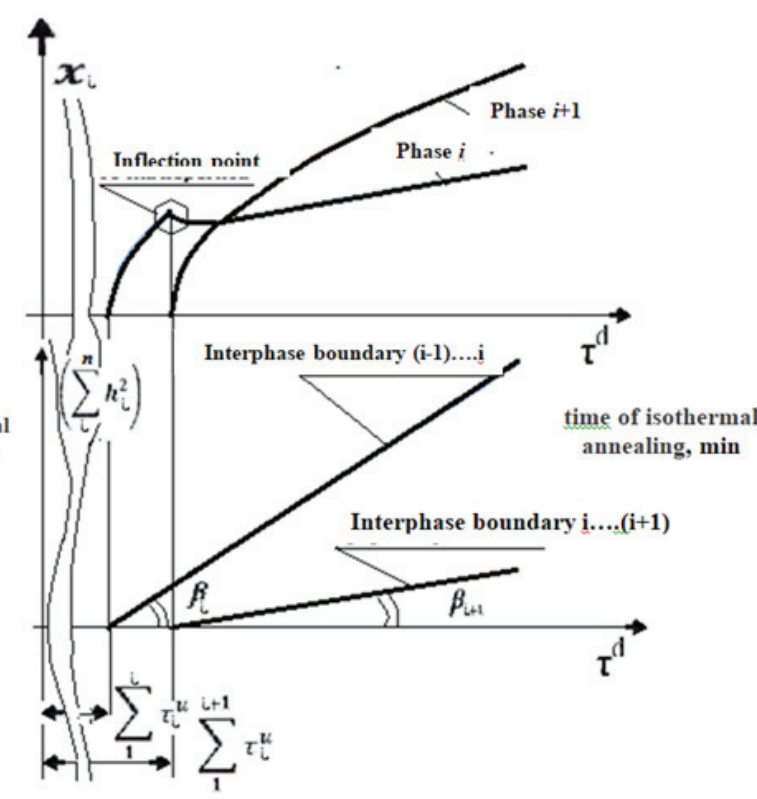

$b$

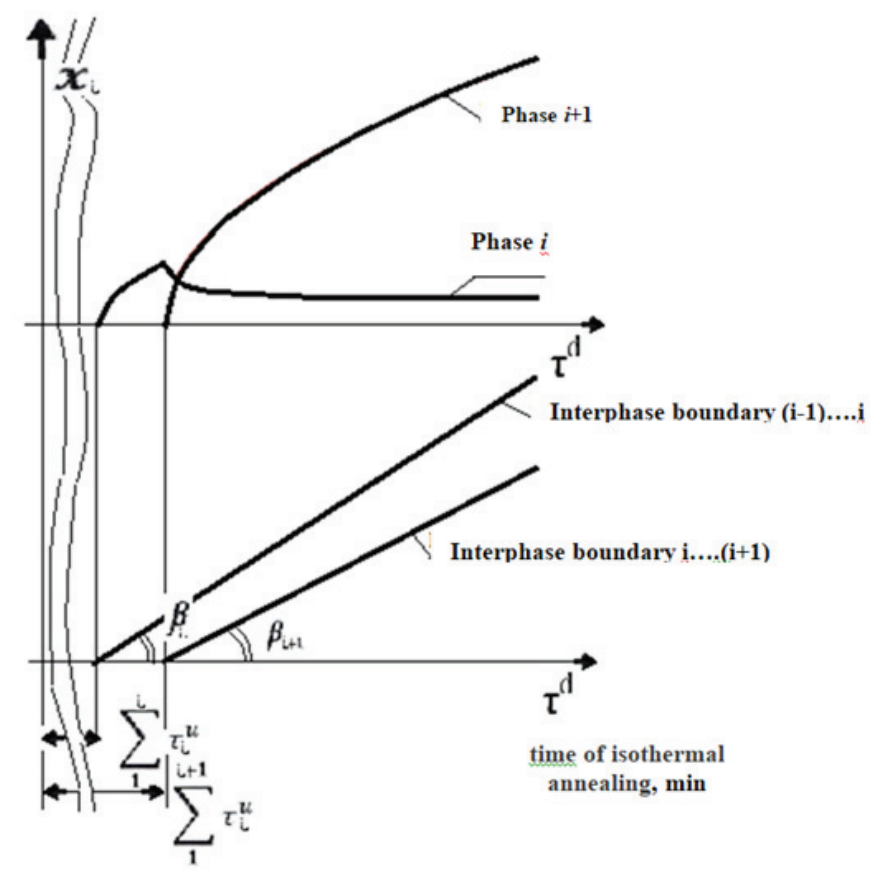

$c$

Рис. 5. Характер роста фазы $i$ и перехода межфазных границ компонентом $A$ в двухкомпонетной многофазной системе при соотношении параметров диффузии $\left(B_{i}>B_{i+1}\right)$ при $\left(\sum_{i}^{u} \tau^{u}<\tau^{d}<\sum_{i+2}^{u} \tau^{u}\right)$

[Fig. 5. The nature of growth of phase $\boldsymbol{i}$ and transition of interphase borders component $A$ in a two-component multiphase system at a ratio of parameters of diffusion of $\left(B_{i}>B_{i+1}\right)$ at $\left.\left(\sum_{i}^{u} \tau^{u}<\tau^{d}<\sum_{i+2}^{u} \tau^{u}\right)\right]$ 

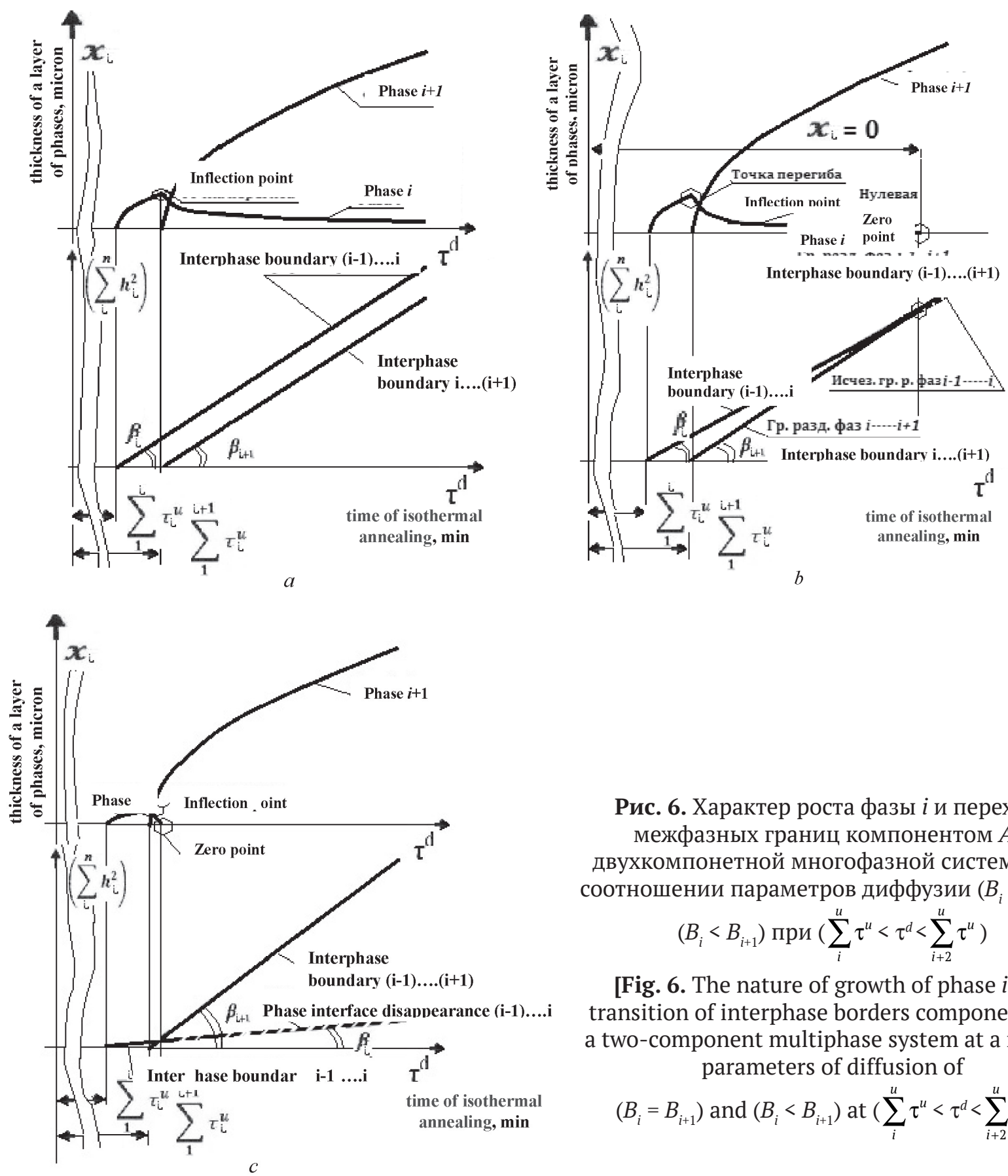

Исследование процессов диффузии крайне важно при разработке технологических процессов сварки, пайки, нанесении покрытий и других, при которых в диффузионной зоне образуются интерметаллические слои. Структура, состав и толщина особенно многофазных слоёв определяют эксплуатационные характеристики материалов, соединений, покрытий и пр. При этом важна не только структура, состав и толщины слоев, образующихся в период их роста, но и изменения, происходящие в диффузионной зоне в период эксплуатации изделий.

Рис. 6. Характер роста фазы $i$ и перехода межфазных границ компонентом $A$ в двухкомпонетной многофазной системе при соотношении параметров диффузии $\left(B_{i}=B_{i+1}\right)$ и

$$
\left(B_{i}<B_{i+1}\right) \text { при }\left(\sum_{i}^{u} \tau^{u}<\tau^{d}<\sum_{i+2}^{u} \tau^{u}\right)
$$

[Fig. 6. The nature of growth of phase $i$ and transition of interphase borders component $\mathrm{A}$ in a two-component multiphase system at a ratio of parameters of diffusion of

$$
\left.\left(B_{i}=B_{i+1}\right) \text { and }\left(B_{i}<B_{i+1}\right) \text { at }\left(\sum_{i}^{u} \tau^{u}<\tau^{d}<\sum_{i+2}^{u} \tau^{u}\right)\right]
$$

Однако описанные в литературе [23-27] различные характеристики роста слоев в диффузионных зонах показывают, что при исследовании процессов диффузии постоянно выявляются новые явления, требующие новых подходов к их научному объяснению, пониманию корреляции между диффузионными и термодинамическими параметрами, методам расчёта и прогнозирования и исследованию понятия «диффузия» в целом. В данных исследованиях обращает внимание противоречивость оценки эффективности того или иного воздействия в процессе диффу- 
зии на развитие физического контакта, активации контактных поверхностей и объёмного взаимодействия.

Для успешного получения новых материалов, их соединений или покрытий с наличием интерметаллидов, карбидов, нитридов, субоксидов, фосфидов и других соединений в технологиях используют разные методы такого воздействия для активизации диффузионных процессов: нагрев, давление, воздействие лазерными или электронными лучами, радиацией, магнитными полями и пр. При этом воздействие может быть осуществлено одновременно несколькими видами внешней энергетической активации в разном сочетании, а также стационарным или циклическим, с медленным нагружением или взрывом. Уровень внешней энергетической активации может затрагивать не только поверхностные слои, но и воздействовать, например, деформировать, на диффузионную зону или всё изделие, приводить к изменению энергии активации диффузионных процессов как внутри самих фазовых слоев, так и на разделяющих их границах. При этом если энергетическое воздействие превышает уровни, вызывающие скачкообразное изменение структуры и свойств как самих материалов, так слоев диффузионной зоны (плавление, дробление зерен, трансформация пластической деформации в другие виды деформации и пр.), скачкообразно будут изменяться и все диффузионные параметры. Но основополагающими будут параметры, наблюдаемые в многофазной системе при уровне внешней энергетической активации, не приводящей к структурным изменениям, как в самих материалов, так и диффузионных слоях.

Поэтому важно при изучении диффузионных процессов в исследуемой системе анализировать отдельно характер роста слоёв для каждого вида внешней энергетической активации. Это позволит более правильно оценить, какое воздействие оказывает на рост слоев каждый вид энергии (температура, давление, радиация, вибрация и пр.), цикличность воздействия и энергетический уровень (ниже или выше уровня структурных изменений как в самих материалах, так в диффузионной зоне и на границах раздела фаз). В результате появляется возможность не только экспериментального определения параметров технологического процесса диффузии, но и их объективная оценка, подтвержденная расчётными данными этих параметров, с целью их оптимизации, а также прогнозирование свойств усовершенствованных или новых изделий в процессе эксплуатации в условиях механического, теплового, радиационного и других видов воздействий (статического, циклического, сложного и т. Д.).

В работе [1] предложена математическая модель роста фаз в двухкомпонентных многофазных системах при изотермическом отжиге. Но эта модель может быть использована и для оценки процессов диффузии изобарных процессов, в условиях радиационного воздействия и пр. При этом, исследование процессов диффузии при одновременном воздействии, например, температуры и давления, позволит оценить вклад каждого вида энергии в соответствии с уравнением (8) и соотношение графиков зависимости $\left(\ln B_{1}=f(1 / T)\right.$ и $\left.\ln B_{2}=f(1 / T)\right)$ для двух соседних фаз по изменению энергии активации при каком-либо одном из указанных видов внешнего воздействия, и при их суммарном воздействии.

\section{ЗАКЛЮЧЕНИЕ}

Результаты настоящего исследования позволяют сделать следующие выводы.

1. При расчетах параметров диффузии необходимо учитывать, что 2-му закону Фика (параболической зависимости от времени диффузии) подчиняется количество компонента, переходящего каждую межфазную границу через единицу площади, а не толщина слоя растущей фазы. Это приводит к более корректному расчету зависимости параметров диффузии с учётом взаимного влияния на них процессов диффузии в двух соседних фазах.

2. Совместный анализ графиков температурной зависимости $\ln B_{1}=f(1 / T)$ и $\ln B_{2}=f(1 / T)$ от температуры отжига для двух соседних фаз: предыдущей $i$ и последующей $(i+1)$ показывает, как изменяется характер роста фазы $i$ после появления на ее границе с компонентом $B$ новой фазы $(i+1)$.

3. Результаты теоретического решения задачи позволяют по известным параметрам температурного режима процесса диффузии, полученным при исследовании двухкомпонентной многофазной системы, целенаправленно контролировать динамику роста, состав образующихся в процессе диффузии слоев, и их выходные параметры в данной системе для получения новых материалов с заданными свойствами.

4. Анализ литературных данных показывает, что множество примеров роста многофазных слоев при изменении температуры про- 
цесса изотермического отжига совпадает с теоретическими построениями, показанными в данной статье.

5. С учётом того, что на процессы диффузии влияют не только температура, но и много других технологических параметров (давление, вибрация и другие виды циклических нагрузок, а также величина и скорость нагружения и пр.) необходимы дальнейшие исследования в данном направлении.

\section{КОНФЛИКТ ИНТЕРЕСОВ}

Авторы декларируют отсутствие явных и потенциальных конфликтов интересов, связанных с публикацией настоящей статьи.

\section{СПИСОК ЛИТЕРАТУРЫ}

1. Molokhina L. A., Rogalin V. E., Kaplunov I. A., Filin S. A. Mathematical model for the growth of phases in binary multiphase systems upon isothermic annealing // Russian Journal of Physical Chemistry A, 2017, v. 91(9), pp. 1635-1641. DOI: 10.7868/ S0044453717090242

2. Molokhina L. A., Rogalin V. E., Kaplunov I. A., Filin S. A. Dependence of growth of the phases of multiphase binary systems on the diffusion parameters // Russian Journal of Physical Chemistry A, 2017, v. 91(12), pp. 2302-2309. DOI: 10.7868/S0044453717120214

3. Лариков Л. Н., Рябов В. Р., Фальченко В. М. Диффузионные процессы в твердой фазе при сварке. М.: Машиностроение, 1975, 192 с.

4. Рослякова Л. И., Росляков И. Н. Диффузионные и кинетические процессы на поверхности стали при цементации // Упрочняющие технологии и покрытия, 2014(4), с. 32.

5. Robinson W. M., Bever M. B. Metallurgical Transactions, 1967, 239, p. 1015.

6. Петрунин И. Е., Маркова И. Ю., Екатова А. С. Металловедение пайки. М.: Металлургия, 1976, 264 c.

7. Иванов С. Г., Гурьев М. А., Гурьев А. М. Расчет коэффициента диффузии процесса одновременного комплексного борирования стали // Актуальные проблемы в машиностроении,2015(2), с. 416420.

8. Гуров К. П., Карташкин Б. А., Угасте Ю. Э. Взаимная диффузия в многофазных металлических системах. М.: Наука, 1981. 350 с.

9. van Loo F. J. J., Rieck G. Diffusion in the Ti-Al system. Interdiffusion between solid $\mathrm{Al}$ and $\mathrm{Fe}$ or Ti-Al alloys // Acta Metallyrg., 1973, v. 21, pp. 61-71. DOI: 10.1016/0001-6160(73)90220-4

10. Борисов В. И., Борисов Т. В. Влияние скорости межфазных реакций на кинетику роста диффузионных слоев // Физика металлов и металловедения, 1976, т. 42, с. 496.
11. Ganseen M., Rieck G. Reactin diffusion and Kirkendall effect in the $\mathrm{Ni}-\mathrm{Al}$ system // Trans. Met. Soc. of AJME, 1967, v. 239, p. 1372.

12. Bastin G. D., Rieck G. Diffusion in the Ti - Ni system. Occurrence and growth of the various intermetallic compounds // Met. Trans. Soc. 1974, v. 5, p. 1817. DOI: $10.1007 /$ bf02644146

13. Clark E. J. Vacuum diffusion joining of titanium // Welding Journel., 1959, v. 38, p. 251.

14. Лашко Н. Ф., Лашко С. В. Вопросы теории и технологии пайки. Саратов, Из-во Саратовского университета, 1976, 248 с.

15. Неверов В. И. Исследование кинетики диффузионного роста фаз в бинарных системах со сложной диаграммой состояния, применяемых в новой технике. Дисс. ... канд. ф.-мат. наук. Свердловск, 1981, 192 с.

16. Бугаков В. З. Диффузия в металлах и сплавах. Ленинград, Гостехиздат, 1949, 206 с.

17. Грызунов В. И., Соколовская Е. М., Айтбаев Б. К. О концентрационной и температурной зависимости коэффициентов диффузии // Изв. АН КазССР. Серия химическая, 1983(6), с. 19-26.

18. Айтбаев Б. К., Грызунов В. И., Соколовская Е. М. Исследование взаимной диффузии в системе титан - цирконий // Вестник Московского универсиmета. Сер. 2, Химия, 1993, т. 34(2), с. 179-180.

19. Гуревич Л. М., Трыков Ю. П., Арисова В. Н., Киселёв О. С., Кондратьев А. Ю., Метелкин В.В. Структура и свойства слоистых титано-алюминиевых композитов, упрочненных частицами интерметаллидов // Известия ВолГТУ. Серия «Проблемы материаловедения сварки и прочности в машиностроении», 2009(59), с. 5-10.

20. Шморгун В. Г., Трыков Ю. П., Слаутин О. В., Богданов А. И, Битюцких А. Е. Влияние термического и силового воздействия на кинетику роста диффузионной прослойки в никель-алюминиевом композите // Известия ВолГТУ. Серия «Проблемы материаловедения сварки и прочности в машиностроении», 2009(59), с. 35-39.

21. Чернышев А. П., Овчинников В. В. Определение инкубационного периода структурных и фазовых превращений в стали // Металловедение и термическая обработка металлов. Известия ВУЗов. Черная металлургия.1998(2), с. 48-49.

22. Treheus G., Guiraldeng P. Infiuence des paliers de reaction isotherme sur la croissance par diffusione des composes d'un diagramme d'equilibre benaire. Compt. Rend. Acad. Sci. B, 1972, v. 275, p. 105.

23. Шмогун В. Г., Трыков Ю. П., Слаутин О. В., Метелкин В. В., Богданов А. И. Кинетика диффузионных процессов в никель-алюминиевой композиции // Известия вузов. Порошковая металлургия и функциональные покрытия, 2008(4), с. 24-28.

24. Мазанко В. Ф., Прокопенко Г. И., Штеренберг А. М., Герцрикен Д. С., Миронова Т. В. Особен- 
ности фазообразования в железе и стали в условиях ультразвуковой ударной обработки // Физика $u$ химия обработки материалов, 2006(2), с. 73-82.

25. Кулемин А. В., Мицкевич А. М. Диффузия в системе $\mathrm{Cu}-\mathrm{Zn}$ при действии знакопеременных напряжений // Металлофизика новейшие технологии, 2007(3), с. 305-315.

26. Крутилин А. Н., Кухарчук М. Н., Сычева О. А. Обзор методов интенсификации диффузионных процессов восстановления оксидов // Литье и металлургия, 2011(60), с. 45-49.

27. Glensk A., Grabowski B., Hickel T., Neugebauer J. Breakdown of the arrhenius law in describing vacancy formation energies: the importance of local anharmonicity revealed by ab initio thermodynamics // Physical Review X, 2014, v. 4(1), p. 011018. DOI: 10.1103/physrevx.4.011018

UDC 544.344.015

DOI: $10.17308 / \mathrm{kcmf} .2019 .21 / 1159$

Received 19.07.2019

Accepted 15.08.2019

\title{
THE ANALYSIS OF INFLUENCE OF TEMPERATURE DEPENDENCE OF PARAMETERS OF DIFFUSION ON THE NATURE OF GROWTH OF LAYERS IN A TWO-COMPONENT MULTIPHASE SYSTEM
}

\author{
(c) 2019 L. A. Molokhina ${ }^{1}$, S. A. Filin ${ }^{2 \bowtie}$ \\ ${ }^{1}$ Kibalchich str., 2, corpus 4, apartment 86, 129164 Moscow, Russian Federation \\ ${ }^{2}$ Plekhanov Russian University of Economics, \\ 36, Stremynny Lane, 117997 Moscow, Russian Federation
}

\begin{abstract}
Purpose. Subject of research - development of phenomenological mathematical model of formation and growth of phases regarding influence of temperature dependence of parameters of diffusion on the nature of growth of layers in a two-component multiphase system, topic-analysis of influence of temperature dependence of parameters of diffusion on change of nature of growth of layers in two-component multiphase systems.

Methods and methodology. Chemical and thermal methods of processing. Novelty of article is the offer of new approach to a scientific explanation of different observed examples of deviations of growth of phases from Fick and Arrhenius's laws and the proof that most of them is connected with lack of accounting of mutual influence on growth of multiphase layers of all phases of a diffusive zone.

Results. It is proposed the solution of a problem of use of temperature condition of process of diffusion when developing technological processes of welding, soldering, application of coverings and others at which in a diffusive zone intermetallic layers, carbides, nitrides, suboxides, phosphides, etc. with the set and controlled operational characteristics of the received new materials, their connections, coverings and so forth are formed.

Conclusions. Results of the solution of a task allow to control purposefully in the known parameters of temperature condition of process of diffusion received at a research of a two-component multiphase system dynamics of growth, structure of the layers which are formed in the course of diffusion, and their output parameters in this system for receiving new materials with the set properties.
\end{abstract}

Keywords: temperature dependence of diffusion, phase, interphase border, multiphase system, mathematical model, component.

$\triangle$ Filin Sergey A., e-mail: Filin.SA@rea.ru 


\section{CONFLICT OF INTEREST}

The authors declare the absence of obvious and potential conflicts of interest related to the publication of this article.

\section{REFERENCES}

1. Molokhina L. A., Rogalin V. E., Kaplunov I. A., Filin S. A. Mathematical model for the growth of phases in binary multiphase systems upon isothermic annealing. Russian Journal of Physical Chemistry A, 2017, v. 91(9), pp. 1635-1641. DOI: 10.7868/ S0044453717090242

2. Molokhina L. A., Rogalin V. E., Kaplunov I. A., Filin S. A. Dependence of growth of the phases of multiphase binary systems on the diffusion parameters. Russian Journal of Physical Chemistry A, 2017, v. 91(12), pp. 2302-2309. DOI: 10.7868/ S00444537171202143.

3. Larikov L. N., Ryabov V. R., Fal'chenko V. M. Diffuzionnye processy $v$ tverdoj faze pri svarke [Diffusive processes in a firm phase when welding]. Moscow, Mashinostroenie Publ., 1975, 192 p. (in Russ.)

4. Roslyakova L. I., Roslyakov I. N. Diffuzionnye i kineticheskie protsessy na poverkhnosti stali pri tsementatsii [Diffusion and kinetic processes on the surface of steel during carburizing]. Uprochnyayuschie tehnologii i pokrytiya, 2014(112), p. 32. (in Russ.)

5. Robinson W. M., Bever M. B. Metallurgical Transactions, 1967, 239, p. 1015.

6. Petrunin I. E., Markova I. Yu., Ekatova A. S. Metallovedenie pajki [Metallurgy Soldering]. Moscow, Metallurgiya Publ., 1976, 264 p. (in Russ.)

7. Ivanov S. G., Gur'ev M. A., Gur'ev A. M. Calculation of diffusion coefficient of simultaneous complex steel borating process. Aktual'nye problemy v mashinostroenii, 2015(2), pp. 416-420. (in Russ.)

8. Gurov K. P., Kartashkin B. A., Ugaste Yu. E. Vzaimnaya diffuziya $v$ mnogofaznyh metallicheskih sistemah [Mutual diffusion in multiphase metal systems]. Moscow, Nauka Publ., 1981, 350 p. (in Russ.)

9. van Loo F. J. J., Rieck G. Diffusion in the Ti-Al system. Interdiffusion between solid $\mathrm{Al}$ and $\mathrm{Fe}$ or Ti-Al alloys. Acta Metallyrg., 1973, v. 21, pp. 61-71. DOI: 10.1016/0001-6160(73)90220-4

10. Borisov V. I., Borisov T. V. Effect of interfacial reaction rate on diffusion layer growth kinetics. Fizika metallov i metallovedeniya, 1976, v. 42, p. 496. (in Russ.)

11. Ganseen M., Rieck G. Effect of interfacial reaction rate on diffusion layer growth kinetics. Trans. Met. Soc. of AJME. 1967, v. 239, p. 1372.

12. Bastin G.D., Rieck G. Diffusion in the Ti-Ni system. Occurrence and growth of the various intermetallic compounds. Met. Trans. Soc. 1974, v. 5, p. 1817. DOI: $10.1007 / \mathrm{bf} 02644146$

13. Clark E. J. Vacuum diffusion joining of titanium. Welding Journel., 1959, v. 38, p. 251.
14. Lashko N. F., Lashko S. V. Pajka metallov [Soldering of metals]. Moscow, Mashinostroenie Publ., 1988,376 p. (in Russ.)

15. Neverov V. I. Issledovanie kinetiki diffuzionnogo rosta faz $v$ binarnyh sistemah so slozhnoj diagrammoj sostoyaniya, primenyaemyh $v$ novoj tehnike [The study of the kinetics of diffusion phase growth in binary systems with a complex state diagram used in the new technique]. Cand. phys. and math. sci. diss. Sverdlovsk, 1981, 192 p. (in Russ.)

16. Bugakov V. Z. Diffuziya v metallah $i$ splavah [Diffusion in metals and alloys]. Leningrad, Gostehizdat Publ., 1949, 206 p. (in Russ.)

17. Gryzunov V. I., Sokolovskaya E. M., Ajtbaev B. K. $O$ kontsentratsionnoy i temperaturnoy zavisimosti koeffitsientov diffuzii [On the concentration and temperature dependence of diffusion coefficients]. Izv. AN KazSSR. Seriya himicheskaya, 1983(6), pp. 19-26. (in Russ.)

18. Ajtbaev B. K., Gryzunov V. I., Sokolovskaya E. M. Issledovanie vzaimnoy diffuzii v sisteme titan tsirkoniy [Study of mutual diffusion in titanium-zirconium system]. Vestnik Moskovskogo universiteta. Ser. 2, Himiya [Moscow University Chemistry Bulletin], 1993, v. 34(2), pp. 179-180. (in Russ.)

19. Gurevich L. M., Trykov Yu. P., Arisova V. N., Kiselev O. S., Kondrat'ev A. Yu., Metelkin V. V. Struktura i svoystva sloistykh titano-alyuminievykh kompozitov, uprochnennykh chastitsami intermetallidov [Structure and properties of layered titanium-aluminum composites reinforced with intermetallide particles]. Izvestiya VolGTU, Seriya «Problemy materialovedeniya svarki i prochnosti v mashinostroenii», 2009(59), pp. 5-10. (in Russ.)

20. Shmorgun V. G., Trykov Yu. P., Slautin O. V., Bogdanov A. I, Bityuckih A. E. Struktura i svoystva sloistykh titano-alyuminievykh kompozitov, uprochnennykh chastitsami intermetallidov \{Effect of thermal and force effects on diffusion layer growth kinetics in nickel-aluminum composite]. Izvestiya VolGTU, Seriya «Problemy materialovedeniya svarki i prochnosti $v$ mashinostroenii», 2009(59), pp. 35-39. (in Russ.)

21. Chernyshev A. P., Ovchinnikov V. V. Opredelenie inkubatsionnogo perioda strukturnykh i fazovykh prevrashcheniy v stali [Determination of incubation period of structural and phase transformations in steel] Metallovedenie i termicheskaya obrabotka metallov. Izvestiya VUZov. Chernaya metallurgiya,1998(2), pp. 48-49. (in Russ.)

22. Treheus G., Guiraldeng P. Infiuence des paliers de reaction isotherme sur la croissance par diffusione des composes d'un diagramme d'equilibre benaire. Compt. Rend. Acad. Sci. B, 1972, v. 275, p. 105.

23. Shmogun V. G., Trykov Yu. P., Slautin O. V., Metelkin V. V., Bogdanov A. I. Kinetika diffuzionnykh protsessov v nikel'-alyuminievoy kompozitsii [Kinetics of diffusion processes in nickel-aluminum composi- 
tion]. Izvestiya vuzov. Poroshkovaya metallurgiya $i$ funkcional'nye pokrytiya, 2008(4), pp. 24-28. (in Russ.)

24. Mazanko V. F., Prokopenko G. I., Shterenberg A. M., Gercriken D. S., Mironova T. V. Osobennosti fazoobrazovaniya $\mathrm{v}$ zheleze i stali $\mathrm{v}$ usloviyakh ul'trazvukovoy udarnoy obrabotki [Features of phase formation in iron and steel under conditions of ultrasonic impact treatment]. Fizika i himiya obrabotki materialov, 2006(2), pp. 73-82. (in Russ.)

25. Kulemin A. V., Mickevich A. M. Diffuziya v sisteme $\mathrm{Cu}-\mathrm{Zn}$ pri deystvii znakoperemennykh napryazheniy [Diffusion in $\mathrm{Cu}-\mathrm{Zn}$ system under al- ternating voltages]. Metallofizika novejshie tehnologii, 2007(3), pp. 305-315. (in Russ.)

26. Krutilin A. N., Kuharchuk M. N., Sycheva O. A. Review of the methods of intensification of diffused processes of oxides deoxidation // Lit'e i metallurgiya, 2011(60), pp. 45-49. (in Russ.)

27. Glensk A., Grabowski B., Hickel T., Neugebauer J. Breakdown of the arrhenius law in describing vacancy formation energies: the importance of local anharmonicity revealed by ab initio thermodynamics. Physical Review X, 2014, v. 4(1), p. 011018. DOI: 10.1103/physrevx.4.011018
Молохина Лариса Аркадьевна - к. т. н., ул. Кибальчича, д. 2, корп. 4, кв. 86, Москва, Российская Федерация; e-mail: lara.molokhina@ mail.ru. ORCID iD 0000-0002-0473-7857.

Филин Сергей Александрович - Д. э. н., к. т. н., доцент, профессор, Российский экономический университет им. Г. В. Плеханова, Москва, Российская Федерация; e-mail: Filin.SA@rea.ru. ORCID iD 0000-0002-6054-6510
Molokhina Larisa A. - Cand. Sci.(Eng.), Kibalchich str., 2, corpus 4, apartment 86, Moscow, Russian Federation; e-mail: lara.molokhina@mail.ru. ORCID iD 0000-0002-0473-7857.

Sergey A. Filin - Dr. Sci. (Econ.), Cand. Sci. (Eng.), Associate Professor, Professor, Plekhanov Russian University of Economics, Moscow, Russian Federation; e-mail: Filin.SA@rea.ru. ORCID iD 0000-0002-6054-6510 